\title{
Catch a moving star
}

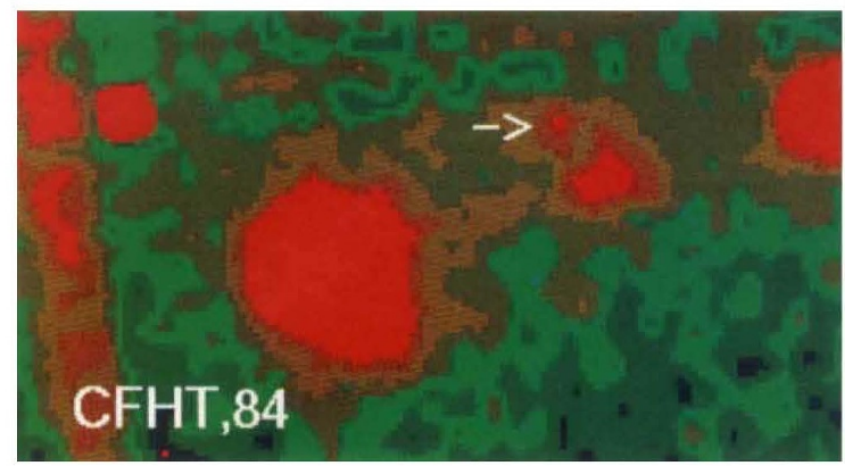

WHEN It became clear last year that the mysterious $\gamma$-ray source Geminga is a $\gamma$-ray pulsar at an apparent distance of less than 400 parsecs, the prospects for measuring its proper motion (perpendlcular to the line of sight) looked promising. On page 704, Blgnaml et al. show that the motion of the putative optlcal counterpart to Geminga, $\mathrm{G}^{\prime \prime}$, can be seen by comparing Images constructed from data from 1984, 1987 and 1992 (the flrst and

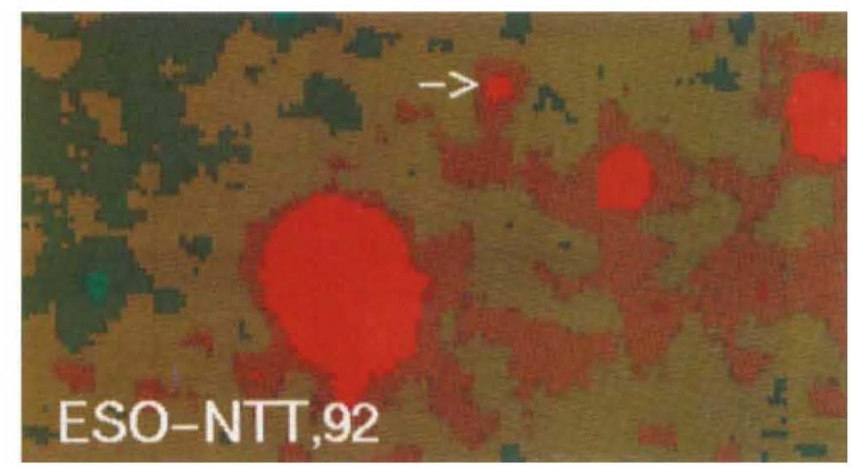

last of which are shown above). The magnitude of the proper motion Is consistent with the Idea that $G^{\prime \prime}$ Is a neutron star (making the association with Geminga Ilkely) about 100 parsecs away. Extrapolation 300,000 years back to the object's position when It was formed In a supernova suggests that thls outburst may have blown the bubble in the Interstellar medium whose orlgin has been a matter for debate (page 706). embryonic development, an interpretation that underscores the surprising nature of the localization of MTase at replication foci. Indeed, why should replication forks be clustered into such foci in the first place?

Part $a$ in the figure, taken from the paper by Leonhardt et al..$^{5}$, shows cells double-labelled with antibodies against the MTase protein (red) and newly synthesized DNA (green). Cells in the G1 or G2 phases of the cell cycle show a diffuse nuclear MTase staining, but $\mathrm{S}$ phase cells, undergoing DNA replication, give a pronounced spotty pattern for both MTase and newly synthesized DNA. The coincidence of the two labels produces a yellow colour. Part $b$ shows sites of DNA replication and part $c$ shows MTase distribution in a single late S-phase nucleus. The two labels are highly co-localized, as shown by part $d$, which is a superposition of $b$ and $c$. While the large toroidal replication foci, which contain centromeric heterochromatin, clearly contain MTase protein, the smaller peripheral foci also stain weakly for MTase. Whether MTase associates with all replication foci to a similar degree, or whether it has a preference for centromeric chromatin is unclear, though co-localization of MTase and replication was observed throughout S phase ${ }^{5}$.

Leonhardt et al. ${ }^{5}$ find that the ability of MTase to localize to replication foci is conferred by a 250 -amino-acid signal sequence at its amino terminus. Targeting does not appear to be required for enzymatic activity, because certain deletions are not targeted (they show a diffuse nuclear staining throughout $S$ phase) but retain full activity with substrates in vitro. This suggests that MTase localization to replication foci is not caused simply by the abundance of hemi- methylated substrate at these sites, but is produced for a specific reason. One likely function that targeting might achieve is to increase the rate of methylation of hemi-methylated sites generated by the replication forks. Increased efficiency for replication-fork proteins by physical association was first suggested for the bacteriophage T4 replication fork, whose component proteins associate into a miniature "protein machine" ${ }^{7}$. But although efficient use of DNA polymerases at a replication fork is required for rapid movement of the fork, it is unlikely that rapid methylation would speed up the process of replication. Further, this does not explain why replication forks (and associated MTases) should be clustered together within the nucleus.

Duplication of the eukaryotic chromosome during the cell cycle requires not only the replication of DNA but the duplication of a number of other features such as DNA methylation, chromatin modification and the presence or absence of transcription factors. Duplication of these chromosomal features is likely to be a sequential process, which might be the key to understanding why MTase is so closely associated with replication foci. Methylation of DNA occurs more rapidly in S-phase cells, possibly because of the relative ease of access of MTase to the DNA when other chromatin proteins are no longer tightly associated with it ${ }^{8}$. Certain proteins can then bind specifically to DNA containing methylated $\mathrm{CpG}$, and this binding seems to be responsible for the repression of transcription that occurs at methylated promoters ${ }^{9,10}$. The presence of such factors is also likely to mediate the degree of overall compaction that the replicated chromatin subsequently undergoes.

The organization of replication forks into clusters may serve to concentrate these sequential processes into functional zones, for instance by allowing DNA methylation to occur before binding of proteins to the nascent DNA. Creation of such zones could also serve to exclude unwanted activities from the region of DNA polymerization; for example, the gapped and unwound DNA structures present at the replication fork might elicit an inappropriate response if detected by DNA repair enzymes or by DNA-binding proteins such as poly(ADP-ribose) polymerase ${ }^{11}$.

The close association of DNA methyltransferase with replication foci emphasizes that the process of chromosome replication in eukaryotic cells is more than the simple duplication of the two strands of DNA, but involves the duplication of other epigenetic features as well. It seems increasingly likely that the spatial organization of replication forks into replication foci plays a part in the co-ordination and control of this sophisticated process.

J. Julian Blow is a Lister Institute Fellow at the ICRF Clare Hall Laboratories, South Mimms, Potters Bar, Hertfordshire EN6 3LD, UK.

1. Nakamura, H., Morita, T. \& Sato, C. Exp. Cell Res. 151 $123-133(1986)$

2. Bravo, R. \& Macdonald-Bravo, H. J. Cell Biol. 105 1549-1554 (1987)

3. Wilcock, D. \& Lane, D. P. Nature 349, 429-431 (1991)

4. Adachi, Y. \& Laemmli, U. K. J. Cell Biol. 119, 1-15 (1992)

5. Leonhardt, H., Page, A. W., Weier, H. \& Bestor, T. H. Cell 71, 865-873 (1992).

6. Li, E., Bestor, T. H. \& Jaenisch, R. Cell 69, 915 (1992)

7. Alberts, B. M. Cold Spring Harbor Symp. quant. Biol. 49, 1-12 (1984)

8. Kastan, M. B., Gowans, B. J. \& Lieberman, M. W. Cell 30, 509-516 (1982).

9. Boyes, J. \& Bird, A. Cell 64, 1123-1134 (1991)

10. Levine, A., Cantoni, G. L. \& Razin, A. Proc. natn. Acad. Sci. U.S.A. 88, 6515-6518 (1991).

11. Satoh, M. S. \& Lindahl, T. Nature 356, 356-358 (1992) 